\title{
Effect of Pharmacist Counseling Intervention on Health Care Utilization Following Hospital Discharge: A Randomized Control Trial
}

Susan P. Bell, MBBS, MSCl',2, Jeffrey L. Schnipper, MD, MPH ${ }^{3}$, Kathryn Goggins, MPH ${ }^{4,5}$, Aihua Bian, MS $S^{6}$, Ayumi Shintani, PhD, MPH', Christianne L. Roumie, MD, MPH 4,5,7, Anuj K. Dalal, MD 3 , Terry A. Jacobson, $M D^{3}$, Kimberly J. Rask, $M D, P h D^{8}$, Viola Vaccarino, $M D, P h D^{8}$, Tejal K. Gandhi, MD, MPH', Stephanie A. Labonville, PharmD ${ }^{10}$, Daniel Johnson, Pharm D ${ }^{11}$, Erin B. Neal, PharmD ${ }^{17}$, and Sunil Kripalani, MD, MSC ${ }^{4,5,12}$ for the Pharmacist Intervention for Low Literacy in Cardiovascular Disease (PILL-CVD) Study Group

\begin{abstract}
'Vanderbilt Center for Translational and Clinical Cardiovascular Research (VTRACC), Division of Cardiovascular Medicine, Department of Medicine, Vanderbilt University, Nashville, TN, USA; ${ }^{2}$ Center for Quality Aging, Division of General Internal Medicine and Public Health, Department of Medicine, Vanderbilt University, Nashville, TN, USA; ${ }^{3}$ Hospitalist Service, Division of General Internal Medicine and Primary Care, Brigham and Women's Hospital and Harvard Medical School, Boston, MA, USA; ${ }^{4}$ Center for Health Services Research, Vanderbilt University, Nashville, TN, USA; ${ }^{5}$ Center for Clinical Quality and Implementation Research, Vanderbilt University, Nashville, TN, USA; ${ }^{6}$ Department of Biostatistics, Vanderbilt University Medical Center, Nashville, TN, USA; ${ }^{7}$ Veterans Health Administration-Tennessee Valley Healthcare System, Geriatric Research Education Clinical Center, Nashville, TN, USA; ${ }^{8}$ Department of Epidemiology, Rollins School of Public Health, and Department of Medicine, School of Medicine, Emory University, Atlanta, GA, USA; ${ }^{9}$ National Patient Safety Foundation, Boston, MA, USA; ${ }^{10}$ Department of Pharmacy Brigham and Women's Hospital and Harvard Medical School, Boston, MA, USA; "Vanderbilt University, Department of Pharmaceutical Services, Vanderbilt University Medical Center, Nashville, TN, USA; ${ }^{12}$ Section of Hospital Medicine, Division of General Internal Medicine and Public Health, Department of Medicine, Vanderbilt University Medical Center, Nashville, TN, USA.
\end{abstract}

BACKGROUND: Reduction in 30-day readmission rates following hospitalization for acute coronary syndrome (ACS) and acute decompensated heart failure (ADHF) is a national goal.

OBJECTIVE: The aim of this study was to determine the effect of a tailored, pharmacist-delivered, health literacy intervention on unplanned health care utilization, including hospital readmission or emergency room (ER) visit, following discharge.

DESIGN: Randomized, controlled trial with concealed allocation and blinded outcome assessors

SETTING: Two tertiary care academic medical centers

PARTICIPANTS: Adults hospitalized with a diagnosis of ACS and/or ADHF

INTERVENTION: Pharmacist-assisted medication reconciliation, inpatient pharmacist counseling, low-literacy adherence aids, and individualized telephone follow-up after discharge

MAIN MEASURES: The primary outcome was time to first unplanned health care event, defined as hospital readmission or an ER visit within 30 days of discharge. Prespecified analyses were conducted to evaluate the effects of the intervention by academic site, health literacy status (inadequate versus adequate), and cognition (impaired versus not impaired). Adjusted hazard ratios (aHR) and $95 \%$ confidence intervals (CI) are reported.

KEY RESULTS: A total of 851 participants enrolled in the study at Vanderbilt University Hospital (VUH) and Brigham and Women's Hospital (BWH). The primary analysis showed no statistically significant effect on time to first unplanned hospital readmission or ER visit among

Clinical Trial Registration: clinicaltrials.gov identifier: NCT00632021

Published online February 16, 2016 patients who received interventions compared to controls (aHR $=1.04,95 \%$ CI 0.78-1.39). There was an interaction of treatment effect by site ( $p=0.04$ for interaction); VUH $\mathrm{aHR}=0.77,95 \% \mathrm{CI} 0.51-1.15 ; \mathrm{BWH}$ aHR $=1.44(95 \% \mathrm{CI}$ 0.95-2.12). The intervention reduced early unplanned health care utilization among patients with inadequate health literacy (aHR 0.41, $95 \%$ CI 0.17-1.00). There was no difference in treatment effect by patient cognition.

CONCLUSION: A tailored, pharmacist-delivered health literacy-sensitive intervention did not reduce postdischarge unplanned health care utilization overall. The intervention was effective among patients with inadequate health literacy, suggesting that targeted practice of pharmacist intervention in this population may be advantageous.

KEY WORDS: Acute coronary syndrome; Heart failure; Readmissions; Health literacy; Pharmacist.

J Gen Intern Med 31(5):470-7

DOI: $10.1007 / \mathrm{s} 11606-016-3596-3$

(c) Society of General Internal Medicine 2016

\section{INTRODUCTION}

Thirty-day readmission rates across the United States following an index admission for acute decompensated heart failure (ADHF) and acute myocardial infarction (AMI) have remained high over the last 10 years with estimated mean risk-standardized readmission rates at $19.9 \%$ for AMI and $24.6 \%$ for ADHF. ${ }^{1}$ Payment penalties linked to readmissions have spurred a focus on reducing 30-day readmissions, ${ }^{2}$ despite limited data for intervention effectiveness. ${ }^{3-6}$ 
As patients transition to home following an acute cardiovascular event, they often receive new and complex medication regimens that are critical to achieving disease improvement and stability. ${ }^{7-9}$ Medication-related problems including discrepancies, non-adherence, and adverse drug events contribute to hospital readmissions. ${ }^{10,11}$ At particular risk are patients with low health literacy (defined as the degree to which individuals have the capacity to obtain, process, and understand basic health information and services needed to make appropriate health decisions) or cognitive impairment, who often have greater difficulty managing medications. ${ }^{12}$

The PILL-CVD (Pharmacist Intervention for Low Literacy in Cardiovascular Disease) study was designed to assess the impact of a tailored, pharmacist-delivered intervention on the number of clinically important medication errors following hospital discharge, health care utilization, and quality of life measures. ${ }^{13}$ Components of the intervention included pharmacist medication reconciliation, inpatient counseling, low-literacy adherence aids, and individualized telephone follow-up after discharge. While the intervention did not demonstrate an overall reduction in medication errors, individuals with low health literacy or impaired cognition were more likely to derive some benefit. ${ }^{14}$ In this follow-up analysis, our aim was to determine if the PILL-CVD intervention had an effect on unplanned health care utilization after hospital discharge and whether this effect was differential by literacy and cognitive status.

\section{METHODS}

\section{Study Design and Setting}

The PILL-CVD Study was a randomized control trial conducted at two academic medical centers - Vanderbilt University Hospital (VUH) in Nashville, Tennessee, and Brigham and Women's Hospital (BWH) in Boston, Massachusetts. Study participants were enrolled in the hospital between May 2008 and September 2009. Participants were randomly assigned to usual care or intervention, and followed for 30 days after discharge. Full details of the PILL-CVD rationale and methods are described elsewhere. ${ }^{13}$ The study was approved by the Vanderbilt University Institutional Review Board and the Partners Human Research Committee.

\section{Participants and Enrollment}

We recruited patients aged 18 and older that were hospitalized for acute coronary syndromes (ACS) and/or acute decompensated heart failure (ADHF), as determined by medical record review conducted by a physician using standard criteria. ${ }^{15,16}$ Key exclusion criteria consisted of severe cognitive impairment (i.e., moderate or severe dementia) or altered mental status, unstable psychiatric illness, inability to communicate in English or Spanish, hospice care, or otherwise being too ill to participate in the baseline interview. Additionally, patients were excluded if they were enrolled in another medication management program, were to be discharged within 3 hours of screening, did not self-manage their medications, or were to be discharged to a location other than home. All participants were enrolled by study research assistants. To avoid biased enrollment, the order in which patients were approached to participate was randomized each day. Termination of the study occurred following complete enrollment and follow-up of the target sample study size.

\section{Baseline Assessment}

After obtaining informed consent, participants completed a series of interviewer-administered measures. Sociodemographic information including age, gender, self-reported race, marital status, insurance status, prior hospital admissions, and primary care provider (PCP) was collected.

The 36-item short form of the Test of Functional Health Literacy in Adults (s-TOFHLA) was administered as an objective measure of health literacy. ${ }^{17}$ Scores can range from 0 to 36 , with higher scores indicating higher health literacy. To assess cognitive impairment, we administered the mini$\mathrm{Cog},{ }^{18}$ which features assessment of delayed recall and executive function. The measure is scored on a scale of $0-5$, where a score of 0-2 indicates cognitive impairment. The four-item Morisky instrument measures pre-hospital medication adherence. ${ }^{19}$ Scores range from $2-8$, with higher scores indicating greater adherence to the medication regimen. After discharge, the hospital length of stay (LOS) and the number of discharge medications were recorded from the electronic medical record.

\section{Randomization and Intervention}

Participants were randomly assigned to receive usual care or intervention in a 1-to-1 ratio. The randomization sequence was computer-generated in permuted blocks of 2-6 patients and was stratified by patient diagnosis and study site. Assignment was managed by a computer program that maintained concealment of treatment allocation and by one unblinded research coordinator at each site who did not play a role in outcome assessment. All investigators, outcome assessors, and biostatisticians were blinded to treatment assignment.

Usual care was in accordance with the Joint Commission requirements. At each hospital, the nurses, pharmacists, and physicians involved in the patients' care performed medication reconciliation and counseling. Post-discharge follow-up calls were not routinely performed in the usual care group.

The study intervention consisted of four specific components. First, pharmacists reconciled preadmission medications and discharge medications with the patient and reported any inconsistencies to the medical team, prior to hospital discharge. Second, the pharmacist provided tailored counseling, which included assessing patient understanding of the medication regimen, barriers to medication adherence, and troubleshooting barriers while the patient was in the hospital. Third, at discharge, the pharmacist provided additional counseling, an 
illustrated medication schedule showing the discharge regimen, and a pillbox, which the patient practiced filling. The pharmacist also employed a teach-back technique to ensure patient understanding. Finally, within four days after hospital discharge, study coordinators contacted the patients and inquired about general health, symptoms, and any medicationrelated problems such as regimen confusion, non-adherence, or side effects. If issues were detected, the study coordinator contacted the pharmacist to provide those patients reinforcement education and to resolve problems.

\section{Unplanned Hospitalizations and ER Visits}

The primary outcome for this analysis was first unplanned health care utilization. This was defined as a composite of first unplanned hospital readmission or ER visit within 30 days after discharge. ER visits that resulted in hospitalization were classified as hospital readmissions. Secondary outcomes included each individual outcome (first unplanned hospital readmission or ER visit). Outcomes were assessed based on a follow-up telephone call with the patient by research staff at approximately 30 days after discharge and by examination of all available medical records. Records were collected from study sites as well as outside facilities.

\section{Statistical Analyses}

The primary analyses evaluated the time to the composite outcome analyzed on an intention-to-treat basis. Individuals who withdrew consent or died during their hospital stay were excluded.
Sample size was calculated based on the primary outcome of the study design (number of clinically important medication errors per patient), and we determined that 862 patients would permit detection of a $30 \%$ reduction in the primary outcome. ${ }^{13}$

The association between intervention and time to first unplanned health care event (hospital readmission or ER visit) was examined using unadjusted and multivariable Cox proportional hazards regression models, and cumulative incidence plots were generated. Models were adjusted for site, age, sex, race (white, non-white), marital status (married/cohabitation, single/living alone), insurance type, presence of PCP, cognitive status, diagnosis (ACS, ADHF, both), length of stay, number of discharge medications, presence of prior hospitalizations and Morisky score (baseline medication adherence). To avoid casewise deletion of records with missing covariates, we employed multiple imputations with five imputation datasets via predictive mean matching. ${ }^{20}$ Less than $1 \%$ of all variables were missing across the dataset. Violations of the proportional hazards assumption were checked graphically.

In addition, as proposed a priori, interaction and sub-group analyses were performed to assess whether the treatment effect was modified by study site, health literacy category, and cognitive status, separately. ${ }^{13}$ Interaction was evaluated by including a cross-product term (treatment group $\mathrm{x}$ each of those variables) in the multivariable Cox proportional hazards regression models. If a significant interaction was detected, a stratified multivariable Cox model was performed.

Statistical significance was determined using a two-sided $5 \%$ significance level $(P<0.05)$. All of the statistical analyses

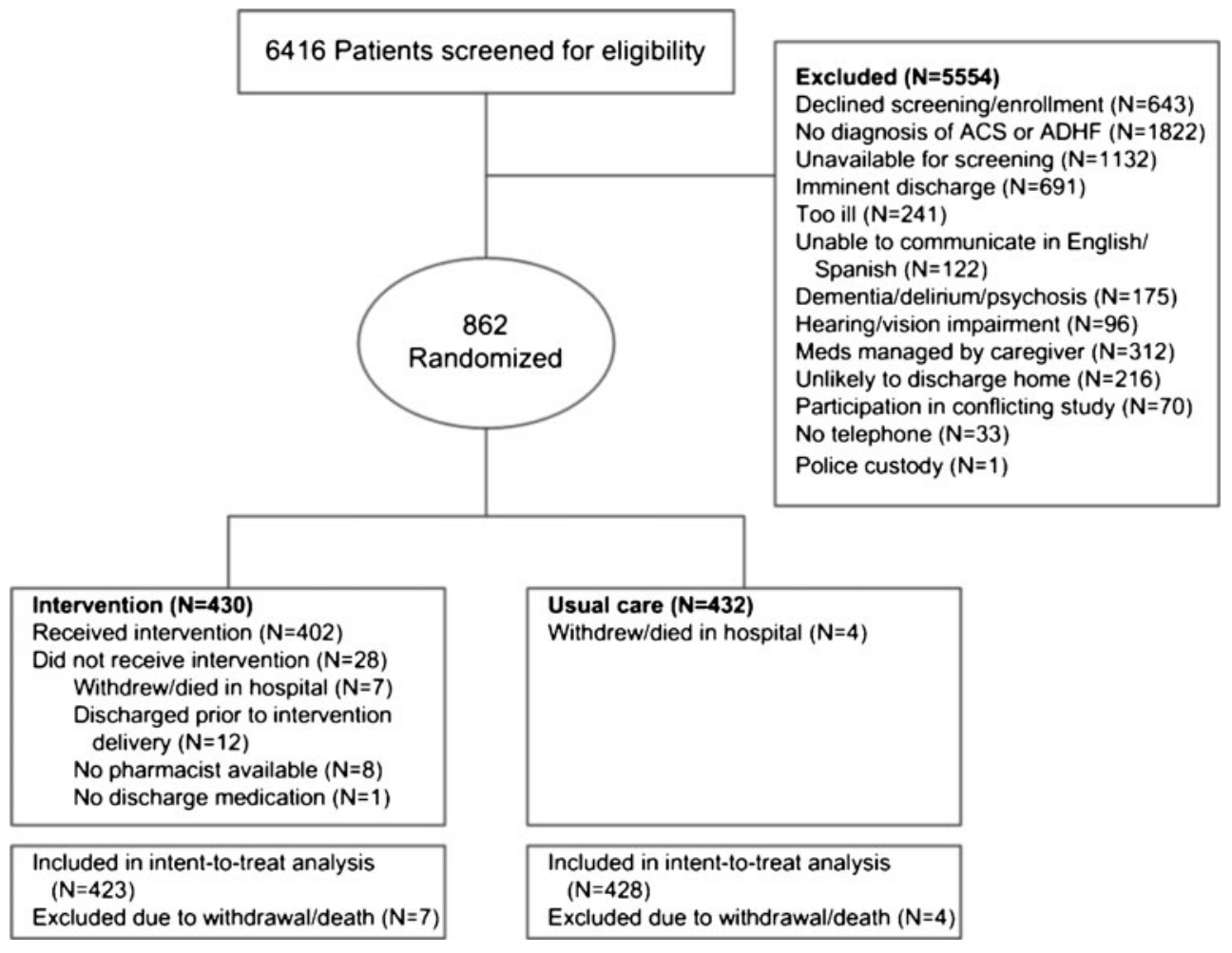

Fig. 1 Study flow diagram 
were performed using R studio, version 3.1.1 (R Development Core Team, Vienna, Austria).

\section{RESULTS}

\section{Participant Selection and Characteristics}

Of 6416 individuals screened for eligibility, 862 patients were randomized (430 to intervention, 432 to usual care) between May 2008 and September 2009. Following randomization, seven intervention patients and four usual-care patients died during hospitalization or withdrew consent. Thus, there were 851 patients of which 423 were intervention (197 VUH and

Table 1 Baseline patient characteristics

\begin{tabular}{|c|c|c|}
\hline Characteristic & $\begin{array}{l}\text { Usual } \\
\text { care } \\
(n=428)\end{array}$ & $\begin{array}{l}\text { Intervention } \\
(n=423)\end{array}$ \\
\hline \multicolumn{3}{|l|}{ Site, N (\%) } \\
\hline Vanderbilt & $200(47)$ & $197(47)$ \\
\hline Brigham & $228(53)$ & $226(53)$ \\
\hline Age, mean years (SD) & $59 \pm 14$ & $61 \pm 14$ \\
\hline Sex, male N $(\%)$ & $249(58)$ & $250(59)$ \\
\hline \multicolumn{3}{|l|}{ Race, N (\%) } \\
\hline White & $335(78)$ & $319(75)$ \\
\hline Black & $71(17)$ & $77(18)$ \\
\hline Other & $18(4)$ & $24(6)$ \\
\hline \multicolumn{3}{|l|}{ Marital status, N (\%) } \\
\hline Married/cohabitating & $256(60)$ & $226(53)$ \\
\hline Single/living alone & $172(40)$ & $197(47)$ \\
\hline \multicolumn{3}{|l|}{ Admission diagnosis, N (\%) } \\
\hline $\mathrm{ACS}$ & $262(61)$ & $260(61)$ \\
\hline $\mathrm{CHF}$ & $133(31)$ & $134(32)$ \\
\hline Both & $33(8)$ & $29(7)$ \\
\hline \multicolumn{3}{|l|}{ Annual income, N (\%) } \\
\hline$<\$ 25,000$ & $107(25)$ & $120(28)$ \\
\hline$\$ 25,000-\$ 49,999$ & $105(25)$ & $103(25)$ \\
\hline$\$ 50,000-\$ 74,999$ & $60(14)$ & $58(14)$ \\
\hline$\geq \$ 75,000$ & $119(28)$ & $105(25)$ \\
\hline \multicolumn{3}{|l|}{ Insurance, $\mathrm{N}(\%)$} \\
\hline Medicare & $162(38)$ & $177(42)$ \\
\hline Medicaid & $37(9)$ & $44(10)$ \\
\hline Commercial & $203(47)$ & $167(39)$ \\
\hline Self-pay/other & $26(6)$ & $35(8)$ \\
\hline Has PCP (N (\%) & $392(92)$ & $386(91)$ \\
\hline \multicolumn{3}{|l|}{ Health literacy (s-TOFHLA) } \\
\hline Total score, Mean (SD) & $30 \pm 7.8$ & $28.9 \pm 8.6$ \\
\hline Inadequate, $\mathrm{N}(\%)$ & $39(9)$ & $47(11)$ \\
\hline Marginal, N (\%) & $38(9)$ & $36(9)$ \\
\hline Adequate, N (\%) & $340(82)$ & $331(80)$ \\
\hline Impaired cognition, N (\%) & $46(11)$ & $52(12)$ \\
\hline \multicolumn{3}{|l|}{$\begin{array}{l}\text { Prevalence of chronic } \\
\text { diseases, N (\%) }\end{array}$} \\
\hline Coronary artery disease & $211(49.3)$ & $225(53.2)$ \\
\hline Cerebrovascular disease & $41(9.6)$ & $30(7.1)$ \\
\hline Hypertension & $296(69.2)$ & $306(72.3)$ \\
\hline Diabetes mellitus & $195(45.6)$ & $140(33.1)$ \\
\hline Hypercholesterolemia & $236(55.1)$ & $234(55.3)$ \\
\hline $\begin{array}{l}\text { Morisky score, Total score } \\
\text { mean, (SD) }\end{array}$ & $6.7 \pm 1.1$ & $6.7 \pm 1.1$ \\
\hline $\begin{array}{l}\text { Number of medications } \\
\text { (discharge), Mean (SD) }\end{array}$ & $8.2 \pm 4.8$ & $9.2 \pm 3.9$ \\
\hline $\begin{array}{l}\text { Length of index } \\
\text { admission, }\end{array}$ & $4.5 \pm 4.6$ & $4.5 \pm 4.1$ \\
\hline Mean days (SD) & & \\
\hline $\begin{array}{l}\text { Hospital admission in } \\
1 \text { year prior, } \mathrm{N}(\%)\end{array}$ & $130(30)$ & $138(33)$ \\
\hline
\end{tabular}

$226 \mathrm{BWH})$ and 428 usual-care (200 VUH and $228 \mathrm{BWH}$ ) (Fig. 1).

The majority of patients were male (59\%) and diagnosed with ACS (61\%); the median age was 60 years, interquartile range (IQR) 51-70 (Table 1). Of particular note, $10 \%$ of the sample had inadequate health literacy, $9 \%$ had marginal, and $81 \%$ had adequate health literacy. There was a high prevalence of chronic comorbidities, and approximately $30 \%$ of individuals had been admitted to hospital in the 12 months before the index hospitalization.

\section{Primary Outcome: Time to First Unplanned Health Care Event}

There were 189 individuals (97 intervention, 92 usual-care) who reached the primary composite outcome of time to unplanned health care utilization during the 30 days following discharge. Analysis of time to first health care event did not show a statistically significant difference between intervention and usual-care patients, adjusted HR=1.04 (95\% CI 0.78 1.39) (Table 2, Fig. 2).

\section{Secondary Outcomes: Readmission or ER Visit Within 30 Days}

The secondary outcome of time to either hospital readmission or ER visit demonstrated no statistically significant difference between intervention and control groups. The adjusted HR for unplanned hospital readmission was 0.94 (95 \% CI 0.63-1.28) and the adjusted HR for ER visits was 1.03 (95\% CI 0.761.39) (Table 2).

\section{Stratified Analyses}

The effect of the intervention on the primary outcome differed when stratified by site (VUH aHR $=0.77,95 \% \mathrm{CI}=0.51$ 1.15; $\mathrm{BWH}$ aHR=1.44, $95 \%$ CI 0.95-2.12), but remained non-significant. Analyses of the secondary outcomes stratified by site were consistent with the main results.

Table 2 Primary and secondary outcomes

\begin{tabular}{|c|c|c|c|c|}
\hline Outcome & $\begin{array}{l}\text { Usual } \\
\text { care } \\
N=428 \\
N(\%)\end{array}$ & $\begin{array}{l}\text { Intervention } \\
N=423 \\
\mathrm{~N}(\%)\end{array}$ & $\begin{array}{l}\text { Unadjusted } \\
\text { HR* (95\% } \\
\text { CI) }\end{array}$ & $\begin{array}{l}\text { aHR** } \\
\text { (95\% \% } \\
\text { CI) }\end{array}$ \\
\hline $\begin{array}{l}\text { Hospital } \\
\text { readmission } \\
\text { or ER visit }\end{array}$ & $\begin{array}{l}92 \\
(21.5)\end{array}$ & 97 (22.9) & $\begin{array}{l}1.09 \\
(0.82-1.45)\end{array}$ & $\begin{array}{l}1.04 \\
(0.78- \\
1.39)\end{array}$ \\
\hline $\begin{array}{l}\text { Hospital } \\
\text { readmission }\end{array}$ & $\begin{array}{l}66 \\
(15.4)\end{array}$ & $61(14.4)$ & $\begin{array}{l}0.94 \\
(0.67-1.34)\end{array}$ & $\begin{array}{l}0.94 \\
(0.63- \\
1.28)\end{array}$ \\
\hline ER visit & $\begin{array}{l}85 \\
(19.9)\end{array}$ & $89(21.0)$ & $\begin{array}{l}1.08 \\
(0.80-1.45)\end{array}$ & $\begin{array}{l}1.03 \\
(0.76- \\
1.39)\end{array}$ \\
\hline
\end{tabular}

*HR represents risk of outcome over 30 days with intervention, using usual-care group as reference, ** Covariate adjustment includes age, sex, race, marital status, insurance type, study site, presence of PCP, cognitive status, diagnosis, length of stay, number of discharge medications, presence of prior hospitalizations, and Morisky score (baseline medication adherence) and health literacy 


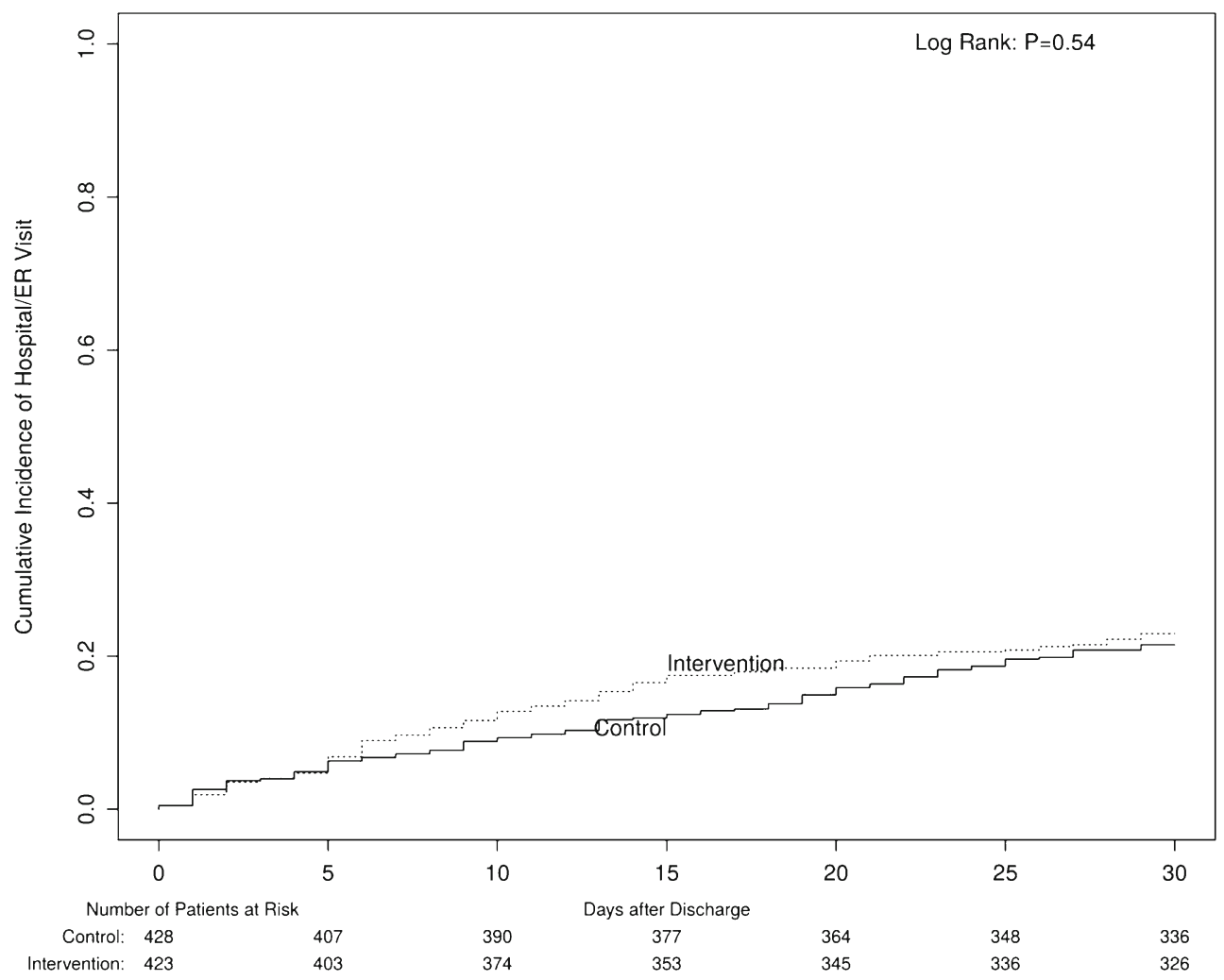

Fig. 2 Cumulative incidence curve of time to first health care utilization event for intervention and control groups over 30 days following discharge

There was a significant interaction of treatment effect with patients' health literacy level ( $p=0.03$ for interaction term). The intervention reduced early, unplanned health care utilization among patients with inadequate health literacy (aHR $=0.41,95 \%$ CI 0.17-1.00), but not among patients with adequate health literacy ( $\mathrm{aHR}=1.07,95 \% \mathrm{CI} 0.77-1.48)$. This effect appeared to be associated with a reduction in early ER visits among those intervention patients with inadequate health literacy $(\mathrm{aHR}=0.29,95 \% \mathrm{CI} 0.11-0.78, P=0.01)$. There was no evidence for an interaction of treatment effect by cognitive status $(P=0.16)$ (Fig. 3$)$.

\section{DISCUSSION}

In this randomized, controlled trial assessing the impact of a tailored, pharmacist-delivered intervention on unplanned health care utilization in the 30 days following hospital discharge, we found no significant difference in the primary outcome of time to first hospital readmission or ER visit. This health literacy-sensitive intervention was effective in reducing unplanned health care utilization among individuals with inadequate health literacy, primarily through an effect on ER visits; this may represent adverse events serious enough to require medical care, but not severe enough to warrant readmission. A differential effect of intervention was also seen by study site, with individuals enrolled at VUH deriving more benefit than those at BWH.
The lack of effect of the intervention overall may reflect the higher than expected literacy levels of our patient population and may also reflect the already high standard of transitional care at these two hospitals. The study was powered to detect a $30 \%$ reduction in the primary outcome of "number of clinically important medication errors per patient"; however, smaller changes in medication errors or resulting health care utilization may have been missed. Our intervention may also have been limited by the use of non-clinical personnel making the initial post-discharge phone call (done to save costs of future broad-based interventions) and the lack of engagement of post-discharge providers in the intervention. Furthermore, the intervention focused on medication-related problems, which are an important cause of unplanned post-discharge health care utilization, but are by no means the only cause. Interventions that have been successful in reducing readmission rates tend to be multifactorial in nature. ${ }^{3,5,6,21}$ While one meta-analysis found no consistent pattern of specific interventions on the efficacy of transitional interventions, ${ }^{4}$ a more recent study found that the more domains of an "ideal transition in care" ${ }^{, 2}$ that were included in the intervention, the more effective it was in reducing readmissions. ${ }^{23}$ Especially important were domains of monitoring and managing symptoms after discharge, enlisting the help of social and community supports, and educating patients to promote self-management.

This educational intervention may have been successful in reducing health care utilization in patients with low health literacy for several reasons. Our intervention provided patient 


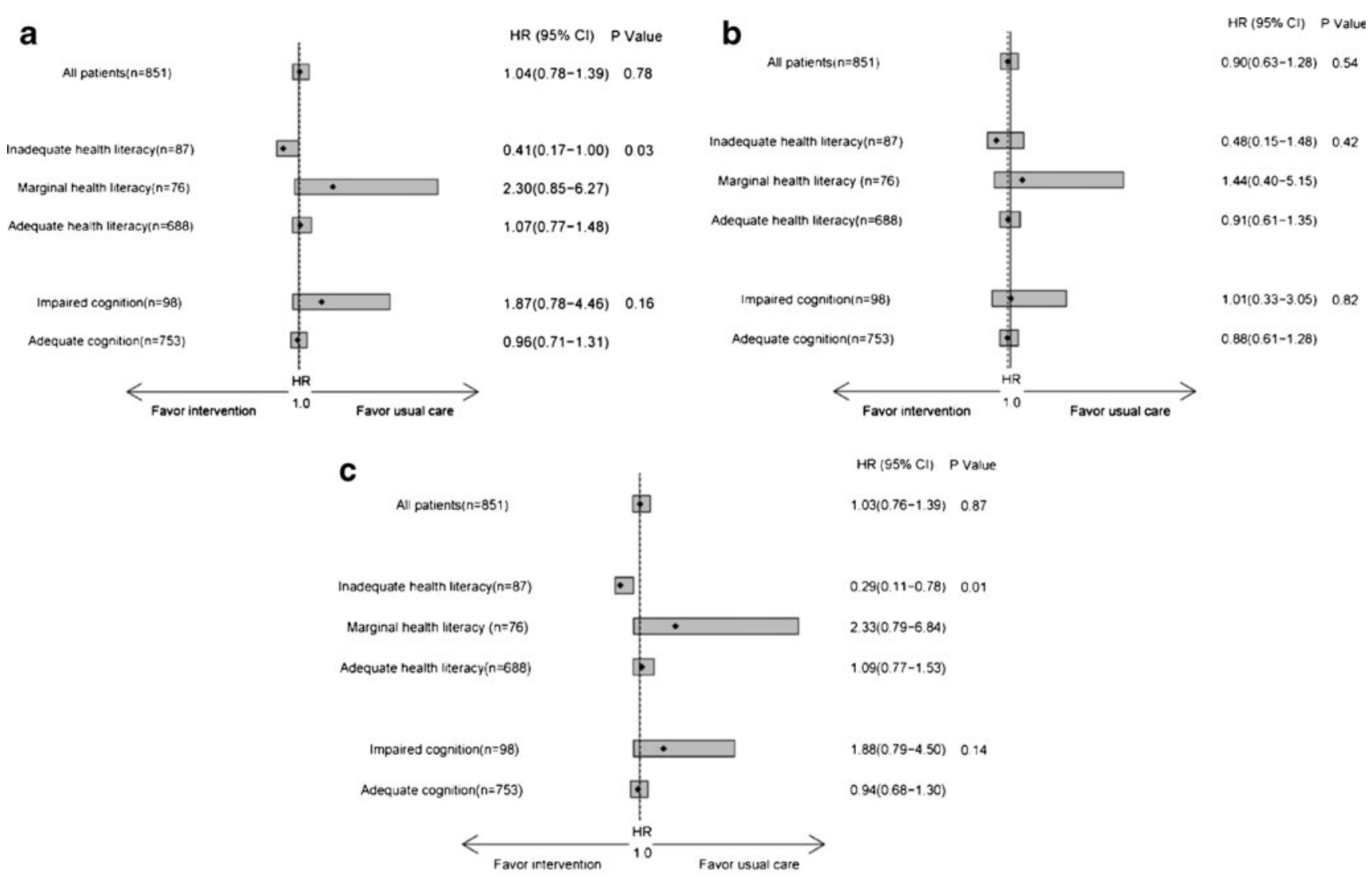

Values less than 1.0 indicate that the mean outcomes in the treatment are smaller than in the usual care group.

Fig. 3 Adjusted treatment effect of intervention on health care utilization by pre-specified sub-group analysis: (a) All events (hospital admission and/or ER visit), (b) Hospital admission, (c) ER visits. Values less than 1.0 indicate that the mean outcomes in the treatment group are smaller than in the control group.

counseling in a low-literacy-friendly manner and sought to bridge a knowledge gap. On average, individuals with lower health literacy often face barriers to understanding the details of safe medication use after discharge and navigating the health care system due to gaps in knowledge, cultural, or educational barriers. This gap may result in medication nonadherence that tends to be unintentional among adults with inadequate health literacy as compared to adults with adequate health literacy. ${ }^{24}$ As we have previously reported, $5.4 \%$ of all patients have an ED visit or are readmitted for medicationrelated issues, ${ }^{14}$ so the closing of this gap may be enough to improve outcomes for this population. On the other hand, patients with adequate health literacy may have fewer barriers to safe post-discharge medication use, or their barriers may be related to attitudes and skills more than knowledge. These deficits may be more difficult to address and require additional forms of intervention. For example, they may require patient coaching, ${ }^{5}$ or more intensive monitoring and management of symptoms through patients' usual sources of care.

Reasons why cognitive function was not an important effect modifier are less clear. Importantly, to participate in the study and informed consent process, patients needed to have relatively intact cognition; patients with moderate or severe dementia were excluded. Those classified as having cognitive impairment in this sample had relatively mild impairment and the effects of caregivers to help with post-discharge care were not assessed in this analysis.

Site differences in the efficacy of the intervention may be multifactorial. The organization of care at the two hospitals was slightly different. BWH has been conducting studies to improve medication reconciliation for years ${ }^{25-27}$ and has fairly robust pharmacist involvement at baseline, such that the incremental benefits of this intervention may have been less. In addition, BWH was a site for implementation of pilot accountable care organization (ACO) that began prior to study enrollment. The patient populations, providers, and health care systems at the two sites are also different in additional ways that were not measured. For example, a greater portion of the patients at Vanderbilt are referred in, live in rural areas, and may have had a different level of access to post-discharge care, and as a result may have benefited more from the additional transitional care services.

The results of this study should be viewed in light of its limitations. First, the study was conducted at two academic medical centers where ongoing, and possibly competing, initiatives to reduce hospital readmissions may have reduced the effect of the intervention and the generalizability of the outcome. Second, the finding that the intervention had a differential affect between sites may also suggest that the impact of the 
intervention may vary across different health care systems with disparate transitional processes of care. The study was powered to demonstrate an overall effect of the intervention regardless of site but not to specifically examine the differential outcomes at BWH and VUH. This does, however, leave open the possibility that although the intervention may not be effective at some hospitals, it may produce a meaningful reduction in health care utilization at others. Future work should look to examine these possibilities in more detail. Finally, the intervention was limited to patients with active cardiovascular disease, so it may not influence care across other disease processes. Care coordination and patient education initiatives, especially prevalent in heart failure programs, may have provided a more robust standard of post-discharge care that may not be available to individuals discharged with alternative primary diagnoses. Additionally, the number of medications and additional confounders specifically affecting subsequent health care utilization following an acute cardiovascular event may not be applicable to all patients discharged from the hospital.

The study has several implications. Hospital-based interventions to improve medication reconciliation and patient knowledge at discharge may have limited efficacy to reduce readmission rates for all patients with CVD. They may be useful among patients, however, with low health literacy. Health literacy stratification may allow sites to focus limited pharmacist resources on those patients most likely to benefit. Screening health literacy levels as part of a standard patient intake evaluation would facilitate this stratification. ${ }^{28}$ Finally, to have a robust effect on post-discharge health care utilization, medication safety interventions may need to be part of multifactorial interventions that focus on several domains of transitional care.

Acknowledgments: The authors wish to thank the following additional individuals for their contributions to the study.

Investigators: David W. Bates, MD, MSc

Biostatistics: Svetlana K. Eden, MSc; Charles Dupont

Research Staff: Courtney Cawthon, MPH; Alexandra Businger; Ileko Mugalla, MS, PhD, MPH; Kurt J. Niesner; Abby G. Meyers, MD; Edith Swain; Jeffrey Kemnitz; Harry Reyes Nieva; Alison C. Pietras; Arianne Cordon, MA; Catherine L. Liang, MPH.

Pharmacists: Judy Cheng, PharmD, MPH; Heather D. Dell'Orfano, PharmD; Radmila Levinson, PharmD; Beth Anne Filkins, PharmD; Pershank Bamarni, PharmD; Eli Guadalupe, DPh; Jill S. Helmke, DPh, $\mathrm{NPh}$; David F. Gregory, PharmD; Marketa Marvanova, PharmD, PhD. Outcome assessors: Kelly Cunningham Sponsler, MD; L. Jeff Harris, MD; Cecelia Theobald, MD, MPH; Robert L. Huang, MD, MPH; Danielle Scheurer, MD, MSc; Susan Hunt, MD.

External advisors: Mark V. Williams, MD; Daniel J. Cobaugh, PharmD.

Corresponding Author: Susan P. Bell, MBBS, MSCI; Vanderbilt Center for Translational and Clinical Cardiovascular Research (VTRACC), Division of Cardiovascular Medicine, Department of Medicine Vanderbilt University, Suite 300-A 2525 West End Avenue, Nashville, TN 37232-8300, USA (e-mail: susan.p.bell@vanderbilt.edu).

\section{Compliance with Ethical Standards:}

Funders: This study was funded by grants R01 HL989755 (SK), K23 HL077597 (SK), and K08 HL072806 (JS) 2K24 HL077506 (VV) from the National Heart, Lung, and Blood Institute. Dr. Bell is supported by K12HD043483-11 from NIH/NICHD and by the Eisenstein Women's Heart Fund.
Prior Presentations: Health Literacy Annual Research Conference, Nov 2014 (poster)

Society of General Internal Medicine Annual Conference, Apr 2015

Conflict of Interest: Dr. Kripalani is a consultant to and holds equity in Bioscape Digital/PictureRx, which makes materials for patient engagement and education. The company's products and services were not used in this study. All other authors declare no potential conflicts of interest

\section{REFERENCES}

1. Krumholz HM, Merrill AR, Schone EM, et al. Patterns of hospital performance in acute myocardial infarction and heart failure 30-day mortality and readmission. Circ Cardiovasc Qual Outcomes. 2009;2:407-13.

2. Patient Protection and Affordable Care Act. Pub L No. 111-148, 124 Stat 408, S305. Hopsitals Readmissions Reduction Program.

3. Burke RE, Coleman EA. Interventions to decrease hospital readmissions: Keys for cost-effectiveness. JAMA Intern Med. 2013;173:695-8.

4. Hansen LO, Young RS, Hinami K, Leung A, Williams MV. Interventions to reduce 30-day rehospitalization: A systematic review. Ann Intern Med. 2011;155:520-8.

5. Coleman EA, Parry C, Chalmers S, Min SJ. The care transitions intervention: Results of a randomized controlled trial. Arch Intern Med. 2006; 166:1822-8.

6. Naylor MD, Brooten D, Campbell R, et al. Comprehensive discharge planning and home follow-up of hospitalized elders: A randomized clinical trial. JAMA. 1999;281:613-20.

7. Kolandaivelu K, Leiden BB, O'Gara PT, Bhatt DL. Non-adherence to cardiovascular medications. Eur Heart J. 2014.

8. Amsterdam EA, Wenger NK, Brindis RG et al. AHA/ACC Guideline for the Management of Patients With Non-ST-Elevation Acute Coronary Syndromes: A Report of the American College of Cardiology/American Heart Association Task Force on Practice Guidelines. J Am Coll Cardiol. 2014.

9. O'Gara PT, Kushner FG, Ascheim DD, et al. ACCF/AHA guideline for the management of ST-elevation myocardial infarction: A report of the american college of cardiology foundation/american heart association task force on practice guidelines. J Am Coll Cardiol. 2013;61:e78-140.

10. Kripalani S, Jackson AT, Schnipper JL, Coleman EA. Promoting effective transitions of care at hospital discharge: A review of key issues for hospitalists. J Hosp Med: Off Publ Soc Hosp Med. 2007;2:314-23.

11. Sokol MC, McGuigan KA, Verbrugge RR, Epstein RS. Impact of medication adherence on hospitalization risk and healthcare cost. Med Care. 2005;43:521-30.

12. Davis TC, Wolf MS, Bass PF 3rd, et al. Literacy and misunderstanding prescription drug labels. Ann Intern Med. 2006; 145:887-94.

13. Schnipper JL, Roumie CL, Cawthon C, et al. Rationale and design of the Pharmacist Intervention for Low literacy in Cardiovascular Disease (PILLCVD) study. Circ Cardiovasc Qual Outcomes. 2010;3:212-9.

14. Kripalani S, Roumie CL, Dalal AK, et al. Effect of a pharmacist intervention on clinically important medication errors after hospital discharge: A randomized trial. Ann Intern Med. 2012;157:1-10.

15. Anderson JL, Adams CD, Antman EM, et al. ACC/AHA 2007 guidelines for the management of patients with unstable angina/non-ST-elevation myocardial infarction: A report of the American College of Cardiology/ American Heart Association task force on practice guidelines (writing committee to revise the 2002 guidelines for the management of patients with unstable angina/Non-ST-elevation myocardial infarction) developed in collaboration with the American College of Emergency Physicians, the Society for Cardiovascular Angiography and Interventions, and the Society of Thoracic Surgeons endorsed by the American Association of Cardiovascular and Pulmonary Rehabilitation and the Society for Academic Emergency Medicine. J Am Coll Cardiol. 2007;50:e1-157.

16. McKee PA, Castelli WP, McNamara PM, Kannel WB. The natural history of congestive heart failure: The Framingham study. N Engl J Med. 1971;285: 1441-6.

17. Baker DW, Williams MV, Parker RM, Gazmararian JA, Nurss J. Development of a brief test to measure functional health literacy. Patient Educ Couns. 1999;38:33-42.

18. Borson S, Scanlan J, Brush M, Vitaliano P, Dokmak A. The mini-cog: A cognitive 'vital signs' measure for dementia screening in multi-lingual elderly. Int J Geriatr Psychiatry. 2000;15:1021-7.

19. Morisky DE, Green LW, Levine DM. Concurrent and predictive validity of a self-reported measure of medication adherence. Med Care. 1986;24:67-74.

20. Rubin DB, Schenker N. Multiple imputation in health-care databases: An overview and some applications. Stat Med. 1991;10:585-98. 
21. Jack BW, Chetty VK, Anthony D, et al. A reengineered hospital discharge program to decrease rehospitalization: A randomized trial. Ann Intern Med. 2009; 150:178-87.

22. Burke RE, Kripalani S, Vasilevskis EE, Schnipper JL. Moving beyond readmission penalties: Creating an ideal process to improve transitional care. J Hosp Med: Off Publ Soc Hosp Med. 2013;8: 102-9.

23. Burke RE, Guo R, Prochazka AV, Misky GJ. Identifying keys to success in reducing readmissions using the ideal transitions in care framework. BMC Health Serv Res. 2014; 14:423.

24. Lindquist LA, Go L, Fleisher J, Jain N, Friesema E, Baker DW. Relationship of health literacy to intentional and unintentional nonadherence of hospital discharge medications. J Gen Intern Med. 2012;27:173-8.
25. Schnipper JL, Hamann C, Ndumele CD, et al. Effect of an electronic medication reconciliation application and process redesign on potential adverse drug events: A cluster-randomized trial. Arch Intern Med. 2009; 169:771-80.

26. Schnipper JL, Kirwin JL, Cotugno MC, et al. Role of pharmacist counseling in preventing adverse drug events after hospitalization. Arch Intern Med. 2006; 166:565-71.

27. Schnipper JL, Liang CL, Hamann C, et al. Development of a tool within the electronic medical record to facilitate medication reconciliation after hospital discharge. J Am Med Inform Assoc: JAMIA. 2011;18:309-13.

28. Cawthon C, Mion LC, Willens DE, Roumie CL, Kripalani S. Implementing routine health literacy assessment in hospital and primary care patients. Joint Comm J Qual Patient Saf / Joint Comm Resour. 2014;40:68-76. 